\title{
Scattering field imaging along an optical waveguide in operando
}

\author{
Yosri Haddad, Jacques Chrétien, Samuel Margueron, Jean-Charles Beugnot, Gil Fanjoux \\ FEMTO-ST institute, University of Franche-Comté, 15B avenue des Montboucons, 25030 Besançon, France
}

The linear or nonlinear optical propagation behavior lengthwise of micro- and nano-waveguides in operation is difficult to evaluate experimentally, although this information is crucial for their optimal development. Previously, apart from the destructive cut-back method, far-field qualitative imaging of Rayleigh scattering (RS) or optical backscatter reflectometry (OBR) technique in infrared wavelength (the state-of-art technique in terms of spatial resolution) have been used for rapid analysis of the propagation of light in optical waveguides (optical nanofiber ONF in Refs. [1, 2]). In the present work, we demonstrate a novel method, nondestructive and noninvasive, with a high spatial (lower than $5 \mu \mathrm{m}$ ) and spectral resolution, to analyze the light propagation in an optical waveguide in operation in linear or nonlinear regime. By measuring with a confocal spectrometer the RS radiated out of an ONF in operation, we show that our method can spatially characterize the entire waveguide in the linear regime with a micrometric spatial resolution. This approach provides direct observation of the higher-order modes (HOMs) leaking from the ONF surface along the taper region and the transition from core-cladding to claddingair guidance. This method can also be used to characterize the linear loss and provides the localization of defects along an optical waveguide.

Figures 1 present experimental results obtained with nanofibers based on a standard single mode fiber SMG652D Fig. 1(a-b) and a SM450 fiber Fig. 1(c), with for both an homogeneous diameter of $1.4 \mu \mathrm{m}$ over $2 \mathrm{~cm}$ long. More precisely, fig. 1(a) shows the backscatter trace from the OBR system at $1566 \mathrm{~nm}$ of wavelength (blue curve) and the scattering trace from our experimental setup (red curve) obtained with a $c w$ laser source at $652 \mathrm{~nm}$ of wavelength. We clearly observe similitudes and differences between these two curves. First, we obtain the same general profile due to the distinguished scattering sources corresponding to the core at the beginning of the nanofiber with large diameter, the cladding when the core does not guide no more, and the surface part when the diameter is very weak [2]. However, large differences are also visible. First, with our method, we clearly observe beating of modes at the beginning of the transition (arrow 1) as well as two successive reduction in the scattering level due to the expansion of different optical modes from core into the cladding region. For lower diameter, we also observe the scattering of the different modes (arrow 2), which are no more guided in the transition part from the standard fiber to the nanofiber. The OBR trace does not show this feature as the fiber is single mode at $1566 \mathrm{~nm}$.
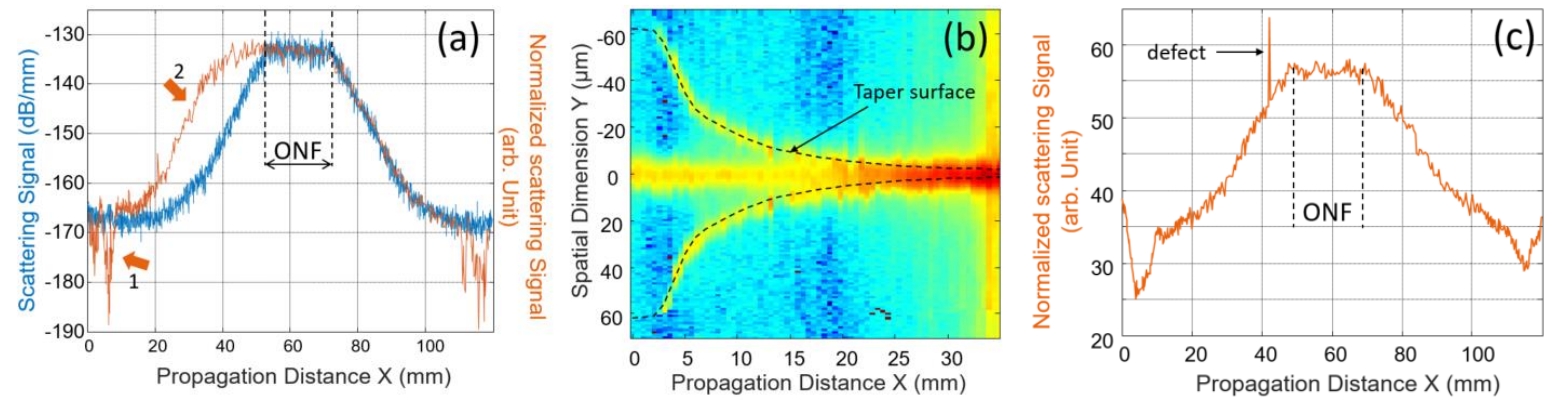

Fig. 1 (a). OBR trace (blue curve) with $\lambda=1566 \mathrm{~nm}$ and trace from our technique (red curve) with $\lambda=652 \mathrm{~nm}$ for a SMFG652D fiber. (b) 2D map of the input transition of a SMFG652D nanofiber in operation. Dotted curve corresponds to the ONF surface. (c) Scattering trace from a SM450 nanofiber, with $\lambda=652 \mathrm{~nm}$.

Our technique has different advantages. First, 2D map of the RS intensity along the waveguide highlight the scattering from the surface at the input transition level (Fig. 1(b)), which comes from the no longer guided HOMs modes, which go out the fiber in the transition 1, and the change of the guiding from core to cladding. The versatility of our technique also allow us to analyze a nanofiber in operando based on a single mode fiber in the visible range SM450 that is impossible to obtain with the OBR measurement (fig. 1(c)). The last advantage of our technique is that we have access to distributed measurements of the nonlinear processes along the fiber due the spectral resolution of our device. We therefore can observe for example the second harmonic generation from quadratic nonlinearity along ridge waveguides, or supercontinuum generation along nonlinear waveguides such as ONF.

\section{References}

[1] J. E. Hoffman et al., "Rayleigh scattering in an optical nanofiber as a probe of higher-order mode propagation", Optica 2, 416-423 (2015)

[2] Y.-H. Lai et al., "Fiber taper characterization by optical backscattering reflectometry", Opt. Exp. 25, 22312 (2017)

[3] A. Godet et al., "Brillouin spectroscopy of optical microfibers and nanofibers", Optica 4, 1232-1238 (2017) 